\title{
In vitro measurement of glucose uptake after radiation and cetuximab treatment in head and neck cancer cell lines using 18F-FDG, gamma spectrometry and PET/CT
}

\author{
NATASA MATIC ${ }^{1,2}$, MARCUS RESSNER ${ }^{3}$, EMILIA WIECHEC ${ }^{1}$ and KARIN ROBERG ${ }^{1,2}$ \\ ${ }^{1}$ Division of Cell Biology, Department of Clinical and Experimental Medicine, Faculty of Health Sciences, \\ Linköping University; ${ }^{2}$ Department of Otorhinolaryngology in Linköping, Anesthetics, Operations \\ and Specialty Surgery Center, University Hospital; ${ }^{3}$ Center for Medical Image Science and Visualization, \\ Linköping University, Linköping, Östergötland SE-58185, Sweden
}

Received April 8, 2019; Accepted August 6, 2019

DOI: $10.3892 / \mathrm{ol} .2019 .10916$

\begin{abstract}
The standard treatment for head and neck squamous cell carcinoma (HNSCC) is radiotherapy, often in combination with chemotherapy or surgery. However, a novel monoclonal antibody, cetuximab (Erbitux ${ }^{\circledR}$ ), has also been approved for patient therapy. The aim of present study was to develop an in vitro method for the measurement of 18F-fluoro-2deoxy-D-glucose (FDG) to determine if cellular $18 \mathrm{~F}-\mathrm{FDG}$ uptake is associated with response to radiotherapy or cetuximab treatment. In the current study, HNSCC cell lines were treated with radiation or with cetuximab. Next, the uptake of 18F-FDG was measured using a gamma spectrometer (GS). Thereafter, uptake after radiation was measured first with GS and then compared with positron emission tomography (PET)/computed tomography (CT) imaging. Furthermore, the mRNA expression of glucose transporter 1 (GLUT1) was measured following cetuximab treatment via reverse transcription-quantitative PCR. A study protocol was developed to measure the cellular uptake of $18 \mathrm{~F}-\mathrm{FDG}$ via gamma-ray spectrometry and comparable results were obtained with those of clinical PET/CT. The results revealed a decrease in $18 \mathrm{~F}-\mathrm{FDG}$ after radiation and cetuximab treatment. The uptake of 18F-FDG following cetuximab treatment was significantly lower in the cetuximab-sensitive cell line UT-SCC-14 compared with the cetuximab-resistant cell lines UT-SCC-2 and UT-SCC-45. Furthermore, after treatment with cetuximab for 24 and $48 \mathrm{~h}$, a significant increase in GLUT1 expression
\end{abstract}

Correspondence to: Professor Karin Roberg, Department of Otorhinolaryngology in Linköping, Anesthetics, Operations and Specialty Surgery Center, University Hospital, Linköping, Östergötland SE-58185, Sweden

E-mail: karin.roberg@liu.se

Key words: head and neck squamous cell carcinoma, radiotherapy, cetuximab, metabolism, 18F-fluoro-2deoxy-D-glucose, glucose transporter 1 was detected in the sensitive cell line compared with the two resistant cell lines. In conclusion, a novel yet reliable method for the measurement of intracellular 18F-FDG via GS has been developed, and our results indicate that 18F-FDG uptake is associated with radiation and cetuximab response in HNSCC.

\section{Introduction}

Head and neck squamous cell carcinoma (HNSCC) originates from the epithelial lining of the oral cavity and is the sixth most common cancer worldwide (1). Modern treatment utilizes a multimodality strategy that primarily involves surgery and radiotherapy and the 5-year overall survival is $\sim 60 \%$. Combinational treatment with chemoradiotherapy, including cisplatin-based chemotherapy, is a standard treatment for locally advanced disease (2). Although molecular targeted drugs such as cetuximab have been developed, they are mostly administered to patients with advanced disease (2). Despite the aforementioned treatment options, resistance and tumor recurrence remain a major concern. The assessment of treatment response is often based on a combination of clinical examinations and functional-anatomical imaging with positron emission tomography (PET), computed tomography (CT) and/or magnetic resonance imaging.

The epidermal growth factor receptor (EGFR) is a transmembrane cell surface receptor that is found mainly in cells of epithelial origin $(3,4)$. The amplification and overexpression of the EGFR gene are frequent events in HNSCC making it a suitable candidate for targeted therapy (3). The monoclonal antibody cetuximab (Erbitux ${ }^{\circledR}$ ) reversibly binds the ligand site (with greater affinity than natural ligands) thereby blocking and preventing the activation of EGFR. This antibody has been approved for the treatment of advanced HNSCC (4).

Altered cell metabolism is a hallmark of cancer and has been shown to negatively influence treatment outcomes in various types of solid tumors including $\operatorname{HNSCC}(5,6)$. Several oncoproteins (such as Akt, Myc and hypoxia-inducible factor $1 \alpha$ ) and oncogenic signaling pathways (such as the P13K/Akt/mTOR pathway) directly regulate important elements of the metabolic network (7). Furthermore, specific 
metabolic signatures have been associated with acquired EGFR tyrosine kinase inhibitor (TKI) resistance and an aggressive growth pattern in HNSCC in vitro and in vivo models (7). Therefore, metabolic imaging serves an important role in translating results from pre-clinical studies to humans. Functional imaging with 18F-fluoro-2-deoxy-D-glucose (FDG) and PET measures metabolic activity in tumors and is an important tool for the staging, treatment planning and follow-up of patients with HNSCC treated with radiation or definitive chemoradiation (8).

FDG is transported into cells via glucose transporters and, of the multiple glucose transporters, glucose transporter 1 (GLUT1) is the most common mediator for glucose uptake (9). This rapid uptake of glucose by GLUT1 promotes cell survival and proliferation and is predominately sustained by hexokinase 2, which is also a regulator of the glucose metabolism (9). GLUT1 is a membrane protein that enables the cellular uptake of glucose through the plasma membrane and has been found to be overexpressed in a number of tumor types, including HNSCC (10-12). Furthermore, in HNSCC EGFR TKI-resistant xenografts, lactate build-up is associated with increased expression of GLUT1 (9).

The identification of cellular prognostic and predictive markers may provide personalized therapeutic cancer treatments (8). Quantitative measurements of changes in cell metabolism could be used to identify markers for treatment response in established cell lines of HNSCC. Therefore, quantitative changes in cell metabolism, together with other cellular characteristics, such as proliferation parameters and metabolic markers, are believed to be a valuable model for the identification of prognosis and treatment response markers (8).

The aim of present study was to develop an in vitro method for measurement of intracellular 18F-FDG with gamma spectrometry and with this, to investigate if $18 \mathrm{~F}-\mathrm{FDG}$ cellular uptake and GLUT1 expression are associated with treatment response in HNSCC cell lines. Furthermore, the potential to compare $18 \mathrm{~F}-\mathrm{FDG}$ uptake in in vitro and in vivo studies via gamma spectrometry and PET/CT may further assist the transition from pre-clinical experiments to early clinical tests and clinical routine to monitor treatment response.

\section{Materials and methods}

Cells and culture conditions. A total of six HNSCC cell lines were used in the current study, three (UT-SCC-2, UT-SCC-14 and UT-SCC-45) cell lines from the University of Turku (gifted by professor Reidar Grenman) and three cell lines (LK0412, LK0626 and LK0858), which were previously established at the University of Linköping (13) (Table I). Normal oral keratinocytes (NOK) were also used. All cell lines were derived from the tissue of patients diagnosed with HNSCC. The cell lines were selected due to their different intrinsic sensitivity for radiation or cetuximab (Table I) $(13,14)$.

The UT-SCC cell lines were cultured in DMEM supplemented with $2 \mathrm{mM}$ glutamine, $1 \%$ non-essential amino acids, $50 \mathrm{IU} / \mathrm{ml}$ penicillin, $50 \mu \mathrm{g} / \mathrm{ml}$ streptomycin and 10\% FBS (all from Gibco; Thermo Fisher Scientific, Inc.). The LK cell lines were cultured in keratinocyte-serum free medium (SFM) supplemented with antibiotics (50 IU/ml penicillin and $50 \mu \mathrm{g} / \mathrm{ml}$ streptomycin) and 10\% FBS (all Gibco; Thermo
Fisher Scientific, Inc.). The cells received fresh culture media twice a week and were subcultured until $\sim 90 \%$ confluence after detaching the cells with $0.25 \%$ trypsin $+0.02 \%$ EDTA. Cultures in passages of 10 to 25 were used in all experiments. Cells were screened periodically for mycoplasma contamination using DAPI staining and/or the Universal Mycoplasma Detection kit (American Type Culture Collection).

NOK cells were established and cultured as previously described (15). Biopsies were harvested during benign surgery in the oral cavity (Department of Otorhinolaryngology, Östergötland) mostly via tonsillectomies and contained non-keratinized squamous cell epithelium (approved by the Linköping University Ethical Committee; diary no. M156-05). Primary keratinocyte cultures were derived from trypsin-digested tissue and cultured in keratinocyte-SFM supplemented with antibiotics $(50 \mathrm{IU} / \mathrm{ml}$ penicillin and $50 \mu \mathrm{g} / \mathrm{ml}$ streptomycin) in culture flasks pre-coated with fibronectin and collagen for $4 \mathrm{~h}$ at $37^{\circ} \mathrm{C}$. Medium was replaced every three days and cells were subcultured to $\sim 75 \%$ confluence using $0.25 \%$ trypsin $+0.02 \%$ EDTA. Cultures were stored at $-150^{\circ} \mathrm{C}$. In the present study, NOK from two patients in passages two and three were used for subsequent analyses.

Assessment of metabolic changes by 18F-FDG measurements. The study protocol was determined after evaluating cellsseeded at a density of 5,000 to 40,000 cells $/ \mathrm{cm}^{2}$ in Petri dishes with an area of $21 \mathrm{~cm}^{2}, 24 \mathrm{~h}$ prior to the administration of 0.5 to 40 megabecquerel (MBq) 18 F FDG solution (incubation times between $15 \mathrm{~min}$ and $3 \mathrm{~h}$ ). The resulting protocol stated below accounts for cell growth, as well as for the saturation of measured intracellular uptake seen in incubation time and administrated activity.

For the experiments, cells were seeded into Petri dishes at densities of $15 \times 10^{3}$ to $30 \times 10^{3}$ cells $/ \mathrm{cm}^{2}$, depending on the plating efficiency of each cell line (70-80\% confluent at the start of the experiments). After cetuximab treatment (24 and $48 \mathrm{~h}$ ) or radiation ( 24 or $72 \mathrm{~h}$ ), cells were starved in PBS for $\sim 1 \mathrm{~h}$, and $1 \mathrm{ml} 18 \mathrm{~F}-\mathrm{FDG}$ solution $(2.0 \pm 0.12 \mathrm{MBq} / \mathrm{ml})$ was added. After $60 \mathrm{~min}$, glucose accumulation was stopped by removing the 18F-FDG solution and thereafter, rinsing the Petri dishes three times with PBS. The intracellular uptake of 18F-FDG was measured using a high purity germanium (HPGe) gamma spectrometer (GS) coupled with a multi-channel analyzer (ORTEC B.V). All cell samples were measured in the same geometry for a real-time acquisition of $120 \mathrm{sec}$, and the registered death time was $<5 \%$ for all measurements. The intracellular uptake of each Petri dish was calculated from the net count of the 511 kiloelectron-volts $(\mathrm{keV})$ photo peak in the spectrum obtained using GammaVision ${ }^{\mathrm{TM}}$ software (7.02.11; ORTEC B.V). The uptake was decay corrected to the time of administration to minimize any time differences between administration and measurement for each batch of cell sample. The remaining intracellular activity in each Petri dish was derived by dividing the total registered number of counts in the photo peak of the acquired gamma spectrum with the acquisition time and the spectrometer source energy peak efficiency factor [1,088 \pm 0.07 counts/kilobecquerel $(\mathrm{kBq})]$. The efficiency factor was obtained from five Petri dish calibration samples of $20 \mathrm{MBq} 18 \mathrm{~F}$ measured in the spectrometer during decay from 3,000 to $25 \mathrm{kBq}$ in the same experimental 
Table I. Origin and tumor characteristics of the investigated cell lines.

\begin{tabular}{|c|c|c|c|c|c|}
\hline Cell line & Primary tumor location & TNM & Sex & IR & ICmabS \\
\hline LK0412 & Tongue & T1N0M0 & $\mathrm{F}$ & 0.63 & \\
\hline LK0626 & Gingiva & T2N0M0 & M & 0.48 & \\
\hline LK0858 & Tongue & T3N0M0 & $\mathrm{F}$ & 0.73 & \\
\hline UT-SCC-2 & Floor of the mouth & T4N1M0 & M & & 0.96 \\
\hline UT-SCC-14 & Tongue & $\mathrm{T} 3 \mathrm{~N} 1 \mathrm{M} 0$ & M & & 0.14 \\
\hline UT-SCC-45 & Floor of the mouth & T3N1M0 & M & & 0.73 \\
\hline
\end{tabular}

TNM, Tumor Node Metastasis classification according to the International Union against Cancer; IR, intrinsic radiosensitivity (surviving fraction at $2 \mathrm{~Gy})$; ICmabS, intrinsic cetuximab sensitivity $(12,13,16)$.

geometry with an overall uncertainty $<3.0 \%$. In addition to the GS measurement, LK626 and LK0412 cell samples in the same conditions as for the GS measurements were stacked and placed in a clinical PET/CT system (Discovery 710; GE Healthcare), as shown in Fig. 1. Images were obtained for one bed position and a bed time of $120 \mathrm{sec}$. Quantification of 18F-FDG uptake was calculated using regions of interest (ROI) over each Petri dish after iterative reconstruction with a standard ordered subsets expectation maximum (OSEM) algorithm of 3 iterations and 18 subsets, Gaussian post filter (full width at half maximum $=5.5 \mathrm{~mm}$ ) and point-spread function (PSF), time-of-flight (TOF) corrections. The quantification steps are illustrated in Fig. S1. The remaining intracellular activity in each Petri dish was derived by dividing the total registered number of counts per sec in the ROI with the acquisition time and the PET-system efficiency factor $(33.47 \pm 1,048$ counts/kBq) derived for this specific measurement. The PET/CT acquisition enables measurements of photon emission per monolayer culture or image voxel to be determined in a single bed for the entire set of Petri dishes and have the possibility to be an accessible method to evaluate treatment sensitivity, drug resistance and therapy response for a large batch in a single acquisition.

The current study assessed the glucose uptake changes in HNSCC cells after radiation and cetuximab treatment, where selected cells were irradiated [4 gray (Gy)] with 4 megaelectron volts photons generated by a linear accelerator (Clinac 4/100; Varian Medical Systems, Inc.), delivering a dose-rate of $2.0 \mathrm{~Gy} / \mathrm{min}$ or treated with cetuximab (Erbitux ${ }^{\circledR} ; 60 \mathrm{nM}$, Merck KGaA). After a further 24 or 48 h, 18F-FDG solution was added and its uptake was measured as described above. The cells were scraped and collected in test tubes, and the protein concentration of each sample was determined using a DC Protein Assay (Bio-Rad Laboratories, Inc.).

Reverse transcription-quantitative PCR (RT-qPCR). The mRNA expression of GLUT1 was analyzed using RT-qPCR on a 7500 Fast Real-Time PCR system (Applied Biosystems; Thermo Fisher Scientific, Inc.). Total RNA was extracted from cells treated with cetuximab (UT-SCC-2, UT-SCC-14 and UT-SCC-45) using the RNeasy mini kit (Qiagen AB) and cDNA was synthesized $\left(37^{\circ} \mathrm{C}\right.$ for $60 \mathrm{~min}, 95^{\circ} \mathrm{C}$ for $5 \mathrm{~min}$ and cooling to $4^{\circ} \mathrm{C}$ ) using the high capacity RNA-to-cDNA kit (Applied Biosystems; Thermo Fisher Scientific, Inc.). TaqMan gene expression assays from Applied Biosystems ${ }^{\circledR}$ (Thermo
Fisher Scientific, Inc) were used for the PCR amplification of GLUT1 (assay ID, Hs00892681_m1; cat. no. 4331182) and two reference genes, GAPDH (assay ID, Hs02758991_g1; cat. no. 4331182) and $\beta$-actin (assay ID, Hs99999903_m1; cat. no. 4331182). The expression levels of GAPDH and $\beta$-actin were used as internal standards. Briefly, the amplification reactions were carried out in $20 \mu \mathrm{l}$ final volume containing $10 \mathrm{ng}$ cDNA, $1 \mu \mathrm{l}$ TaqMan gene expression assay, $10 \mu \mathrm{l}$ TaqMan gene expression master mix and water. The PCR conditions were as follows: $95^{\circ} \mathrm{C}$ for $10 \mathrm{~min}$, followed by 40 cycles of $95^{\circ} \mathrm{C}$ for $15 \mathrm{sec}$ and $60^{\circ} \mathrm{C}$ for $60 \mathrm{sec}$. The comparative $\mathrm{Cq}$ method was applied to determine the $n$-fold difference in expression levels relative to calibrator sample (untreated cells) (16).

Statistical analysis. All data are expressed as the mean \pm SD from three or more independent experiments. Data were statistically evaluated using one-way ANOVA followed by Bonferroni's post hoc test. IBM SPSS statistics v22 (IBM Corporation) was used for all statistical analyses. $\mathrm{P}<0.05$ was considered to indicate a statistically significant difference.

\section{Results}

Quantitative measurements of glucose uptake with FDG. To establish a method for quantitative measurements of glucose uptake in cultured HNSCC cells and NOKs, the radiolabeled glucose analog, 18F-FDG, was administrated to cells and its intracellular uptake was measured via gamma spectrometry. The study protocol was developed in order to maximize the glucose uptake differences between different cell lines, as well as between radiated cells and non-radiated controls. To establish a protocol, different parameters including starvation, administrated activity (0.5-40 MBq 18F-FDG solution), incubation time and rinsing time were investigated for cell line LK0412. The results (data not shown) demonstrated that the uptake of 18F-FDG increased linearly with cell number and administered activity. However, the number of counts per $\mathrm{MBq}$ at a cell line specific density reached a saturation level for administered activity above $2 \mathrm{MBq} /$ Petri dish due to dead time limitations of the spectrometer. The uptake was highest during the first $60 \mathrm{~min}$ of incubation and the saturation level was reached after 60 to $120 \mathrm{~min}$ depending on the number of the cells. The $\sim 60 \mathrm{~min}$ incubation time also corresponds to the well-established standard waiting time between injection of 


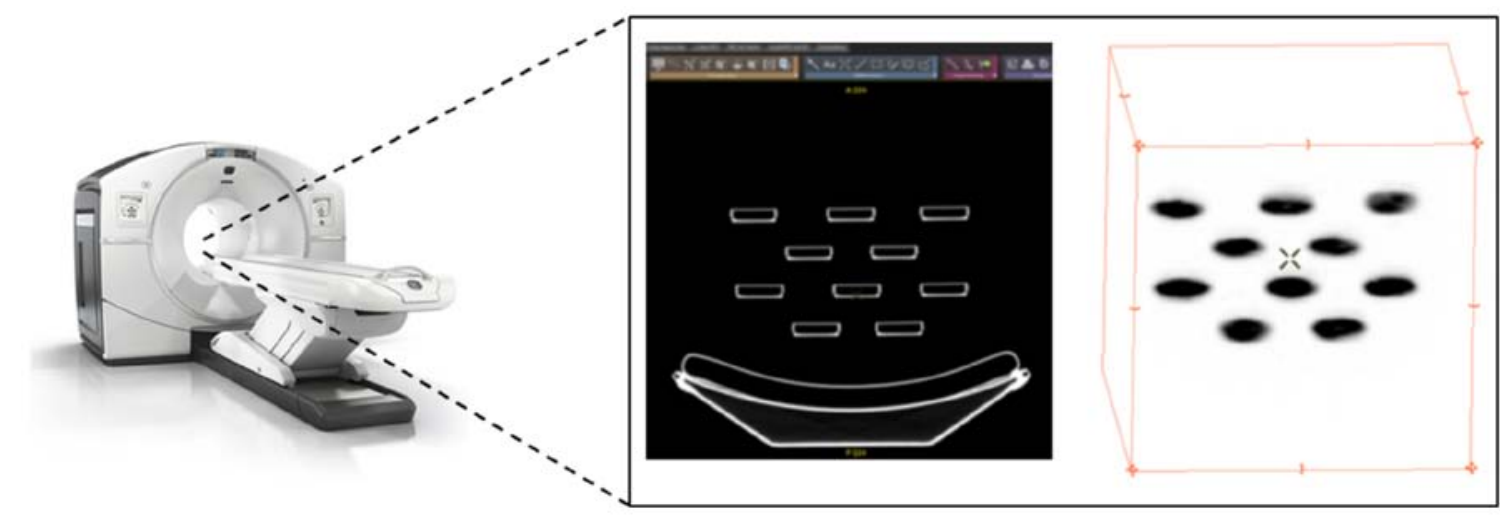

Figure 1. Measurement of 18F-FDG intracellular uptake in cell monolayers grown in Petri dishes and placed in a clinical PET/CT scanner. The CT image (left) and PET image (right) of a batch containing ten cell cultures. 18F-FDG, 18F-fluoro-2deoxy-D-glucose; PET, positron emission tomography; CT, computed tomography.

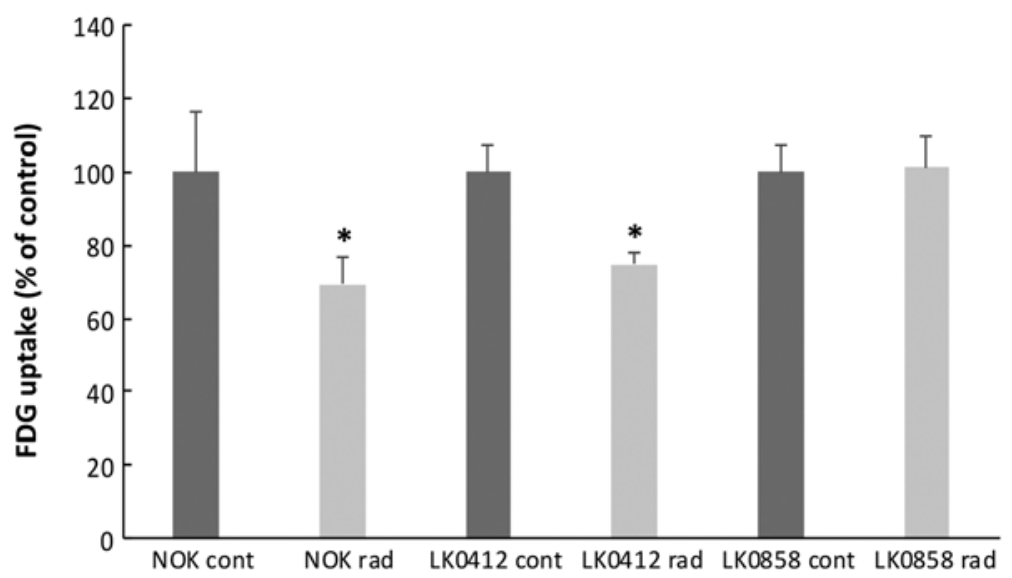

Figure 2. Quantification of glucose uptake following radiation of two head and neck squamous cell carcinoma cell lines (LK0412 and LK0858) and NOKs. Cells were cultured for $24 \mathrm{~h}$ prior to radiation treatment with $4 \mathrm{~Gy}$, and $72 \mathrm{~h}$ later, $2 \mathrm{MBq} 18 \mathrm{~F}-\mathrm{FDG}$ was added for 60 min. Values represent intracellular activity (FDG uptake)/mg protein relative to controls (mean $\pm \mathrm{SD} ; \mathrm{n}=5$ ). A significant decrease in FDG uptake was measured following radiation in the radiosensitive cell line LK0412 and in NOK compared with untreated cells. Data were statistically evaluated using one-way ANOVA followed by Bonferroni's adjustment; "P<0.05 vs. control. NOKs, normal oral keratinocytes; 18F-FDG, 18F-fluoro-2deoxy-D-glucose; cont, control; rad, radiation; MBq, megabecquerel; Gy, gray.

18F-FDG and PET image acquisition for clinical PET oncology examinations. The favored protocol consisted of a starvation time with PBS of 1-2 h, a cell line specific cell-seeding, an administrated activity of $2.0 \mathrm{MBq} 18 \mathrm{~F}-\mathrm{FDG}$ in $1 \mathrm{ml}$ of PBS and an incubation time of $60 \mathrm{~min}$.

Differences in glucose uptake in HNSCC after radiation. To investigate if the radiation sensitivity of tumor cells and NOKs could be predicted by measuring changes in glucose uptake, the radiolabeled glucose analog F18-FDG was quantitatively analyzed via gamma spectrometry before and $72 \mathrm{~h}$ after radiation treatment (4 Gy). HNSCC cell lines LK0412 and LK0858 with different radiosensitivities (Table I; surviving fraction at 2 Gy) (13) and NOKs were investigated. A significant decrease in metabolic activity was measured after radiation in the more radiosensitive cell line, LK0412 and in NOK (Fig. 2). No differences in morphology were observed between control cells and radiated cells (data not shown) and all data were normalized to the amount of protein in each Petri dish.

Differences in glucose uptake in HNSCC after treatment with cetuximab. To investigate if cell sensitivity to cetuximab could be predicted by measuring intracellular glucose uptake, F18-FDG was quantitatively analyzed via gamma spectrometry. The dose of cetuximab $(60 \mathrm{nM})$ was chosen from previous experiments $(14,17)$. The glucose uptake of UT-SCC-14, UT-SCC-45 and UT-SCC-2 HNSCC cell lines with different cetuximab sensitivities $(14,17)$ (Table I) was assessed before and after 24 and $48 \mathrm{~h}$ of cetuximab treatment. After $24 \mathrm{~h}$, a significantly decreased uptake of F18-FDG was determined in the most sensitive cell line UT-SCC-14, when compared with the most resistant cell line, UT-SCC-2. Furthermore, significant differences were also observed between UT-SCC-2 (24 h) cells and the middle sensitive cell line, UT-SCC-45 (Fig. 3); no significant differences were observed after $48 \mathrm{~h}$.

Changes in GLUT1 mRNA expression after cetuximab treatment. To investigate the association between GLUT1 expression and F18-FDG uptake in cell lines possessing diverse intrinsic sensitivities to cetuximab (UT-SCC- 2, UT-SCC-14 and UT-SCC-45), the mRNA expression of GLUT1 was determined via RT-qPCR prior to and following $60 \mathrm{nM}$ cetuximab treatment. After 24 and 48 h cetuximab treatment, the expression of GLUT1 was significantly increased in UT-SCC-14 


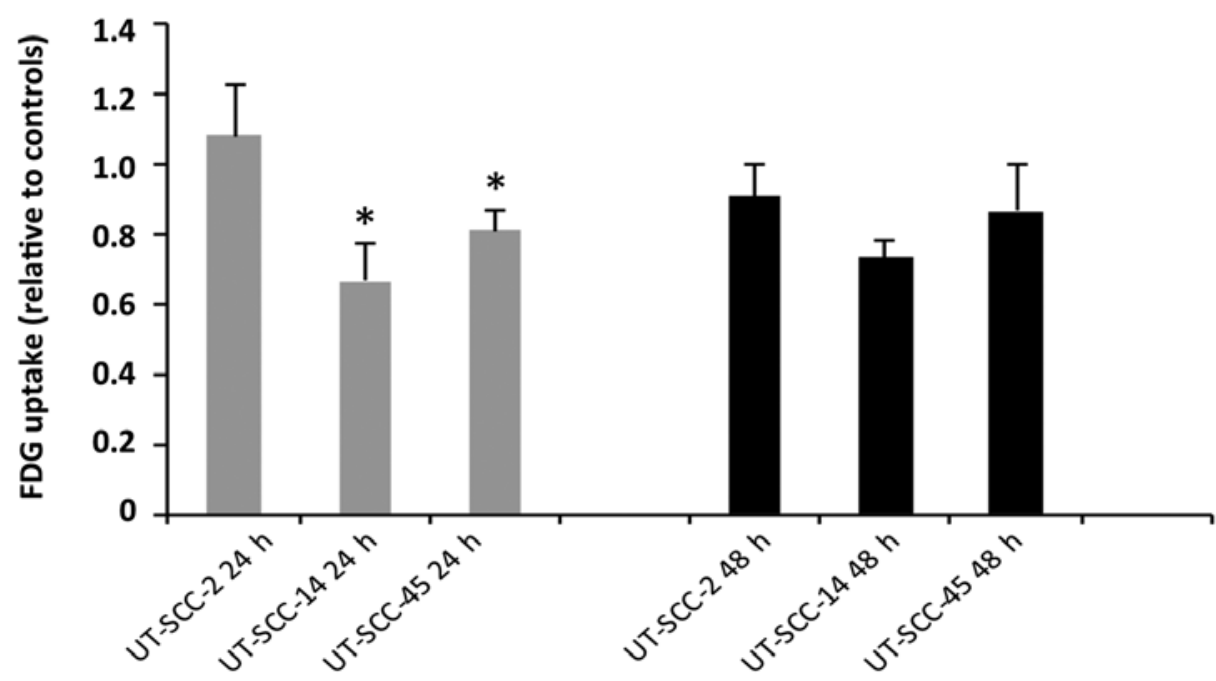

Figure 3. Quantification of glucose uptake in untreated and cetuximab treated head and neck squamous cell carcinoma cell lines (UT-SCC-2, UT-SCC-14 and UT-SCC-45). Cells were cultured for $24 \mathrm{~h}$ prior to treatment with cetuximab $(60 \mathrm{nM})$. and 24 or $48 \mathrm{~h}$ later, $2 \mathrm{MBq}$ 18F-FDG was added for $60 \mathrm{~min}$, and intracellular activities were measured with a high purity germanium solid state photon detector. Values represent intracellular activity (FDG uptake)/mg protein relative to controls (mean $\pm \mathrm{SD} ; \mathrm{n}=5$ ). Following 24-h cetuximab treatment, a significantly decreased uptake of F18-FDG was observed in the most sensitive cell line UT-SCC-14 and in the moderately sensitive cell line UT-SCC-45 compared with the most resistant cell line UT-SCC-2. Data were statistically evaluated using one-way ANOVA followed by Bonferroni's adjustment; "P<0.05 vs. UT-SCC-2. 18F-FDG, 18F-fluoro-2deoxy-D-glucose; MBq, megabecquerel.

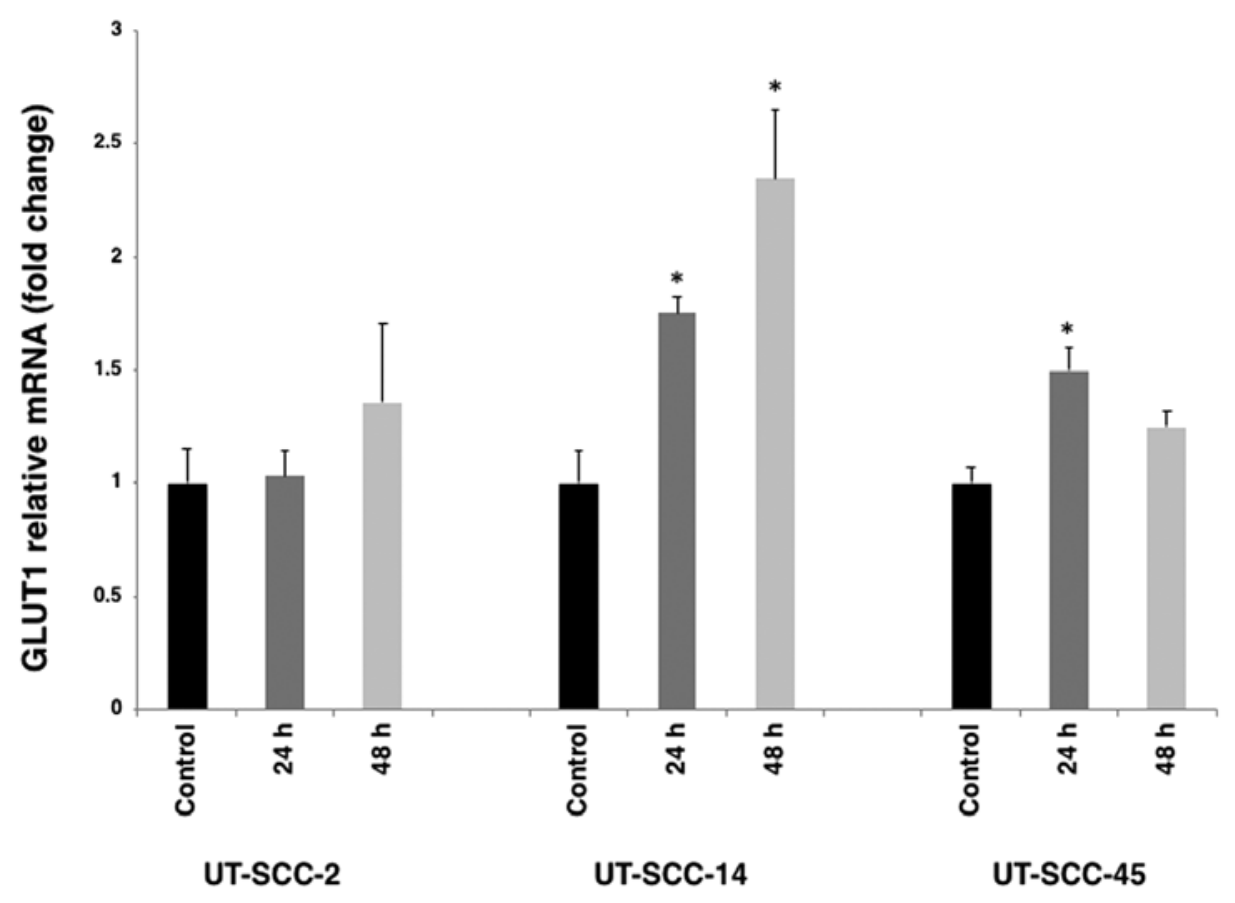

Figure 4. mRNA expression of GLUT1 in head and neck squamous cell carcinoma cells (UT-SCC-2, UT-SCC-14 and UT-SCC-45) following exposure to $60 \mathrm{nM}$ cetuximab for 24 and $48 \mathrm{~h}$. The expression of GLUT1 was significantly increased following 24- and 48-h cetuximab treatment in UT-SCC-14 and, to a lesser extent, UT-SCC-45 cells compared with untreated control cells. Data were normalized to untreated cells for each cell line (mean \pm SD; $n=3$ ). Data were statistically evaluated using one-way ANOVA followed by Bonferroni adjustment; "P<0.05 vs. control. GLUT1, glucose transporter 1.

and to a lesser extent in UT-SCC-45 cells. Moreover, after 24 and $48 \mathrm{~h}$ of cetuximab treatment, differences in GLUT1 mRNA expression pattern were identified between the cell lines (Fig. 4).

Comparison between gamma spectrometry and PET measurement of FDG uptake. The quantification of glucose uptake for LK0412 and LK0626 HNSCC cell lines with different radiation sensitivities (13) was compared via gamma spectrometry and PET/CT imaging to estimate early response $(24 \mathrm{~h})$ after radiation (4 Gy). The source full energy peak efficiency factor (counts/kBq) was derived for both the PET-system and the HPGe gamma detector by measuring the counts in the photo peak $511 \mathrm{keV}(\mathrm{PET} \pm 10 \%$; HPGe $\pm 1 \%$ ) from an in-house calibrated sample in a fixed geometry during decay from $3 \mathrm{MBq}$ to $25 \mathrm{kBq}$.

Each method was able to distinguish the relative difference in $18 \mathrm{~F}-F D G$ uptake between the two cell lines. The mean 


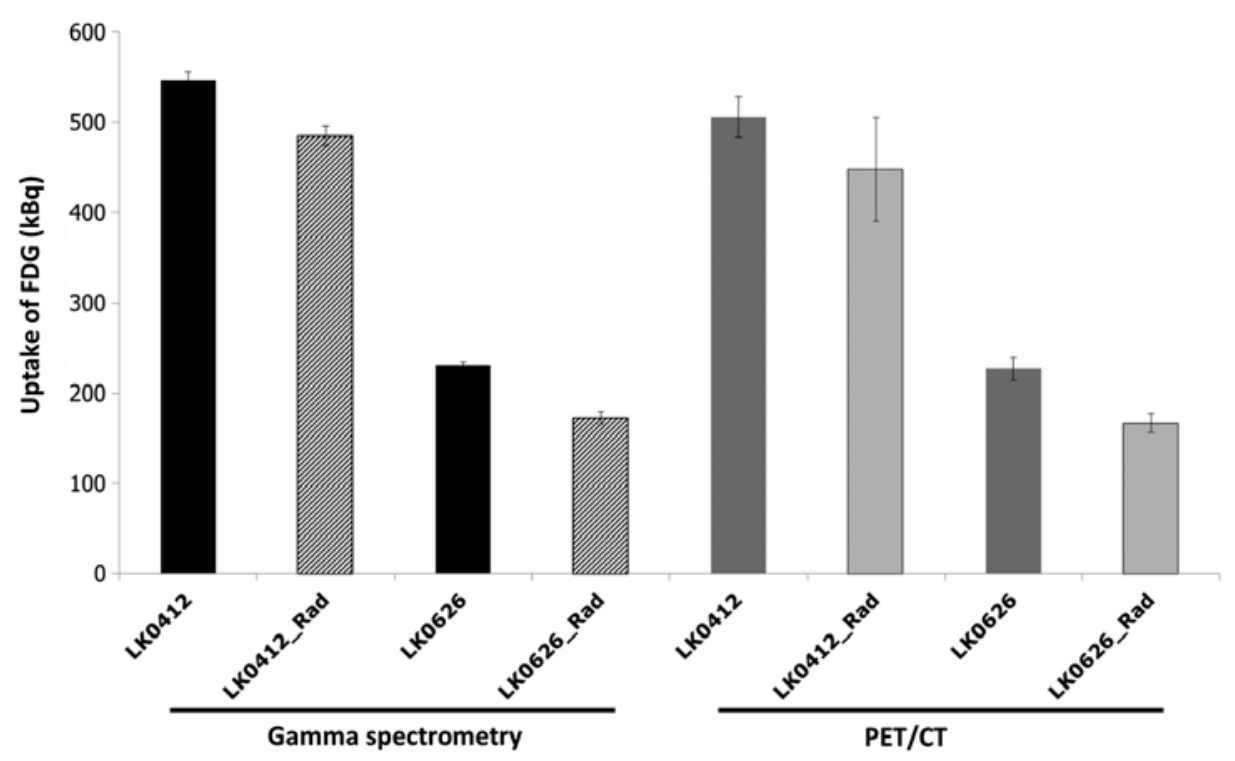

Figure 5. Intracellular uptake of 18F-FDG in head and neck squamous cell carcinoma cell lines (LK0412 and LK0626), as determined via gamma spectrometry (black) and PET/CT (gray). Cells were cultured for $24 \mathrm{~h}$ prior to radiation treatment with $4 \mathrm{~Gy}$ and cultured for an additional $24 \mathrm{~h}$ prior to the addition of $2 \mathrm{MBq}$ 18F-FDG for $60 \mathrm{~min}$, intracellular activities were measured with a high purity germanium solid state photon detector and a clinical PET/CT system. The values were decay corrected to the reference time of $18 \mathrm{~F}-\mathrm{FDG}$ administration. The graph represents one of two experiments with 5 Petri dishes in each group (mean $\pm \mathrm{SD}$; $\mathrm{n}=5$ ). 18F-FDG, 18F-fluoro-2deoxy-D-glucose; PET, positron emission tomography; CT, computed tomography; Rad, radiation; $\mathrm{kBq}$, kilobecquerel.

difference uptake and standard deviation was $280 \pm 39.5 \mathrm{kBq}$ (PET-system) and $314 \pm 11.4 \mathrm{kBq}$ (HPGe detector) for controls and radiated samples obtained subsequently under same conditions (Fig. 5). For the more radiation-sensitive cell line LK0626, the two methods could clearly identify the irradiated dishes from the controls (GS, non-irradiated $231 \pm 3.4 \mathrm{kBq}$ and irradiated $172 \pm 7.2 \mathrm{kBq}$; and $\mathrm{PET} / \mathrm{CT}$, non-irradiated $227 \pm 13.0 \mathrm{kBq}$ and irradiated $166 \pm 10.2 \mathrm{kBq}$ ) (Fig. 5). For the less sensitive cell line LK0412, the spectrometry measurement was more sensitive with less variation (non-irradiated $546 \pm 9.2 \mathrm{kBq}$ and irradiated $485 \pm 10.3 \mathrm{kBq}$ ) compared with the PET/CT measurement (non-irradiated $505 \pm 22.6 \mathrm{kBq}$ and irradiated $447 \pm 57.3 \mathrm{kBq}$ ) (Fig. 5).

\section{Discussion}

Cancer cells require a large quantity of glucose for growth and proliferation, and changes in cell metabolism are thought to impact treatment response but to various extents in different types of tumor. The assessment of glucose metabolism may represent a method to determine intracellular metabolic activity. Increased uptake of FDG observed in mammary tumor cells has been revealed to directly indicate a higher glucose metabolic rate (18). Furthermore, high uptake of FDG has been associated with poor prognosis (19). The main aim of the current study was to develop a reliable method for measuring 18F-FDG cellular uptake. Therefore, a method for the in vitro measurement of glucose uptake was established using a gamma spectrometer. Whether the intracellular uptake of $18 \mathrm{~F}-\mathrm{FDG}$ could be used as a reliable predictive marker for radiotherapy and cetuximab responses was determined.

Gamma spectrometry is a non-invasive technique that is used to measure electromagnetic radiation in the gamma spectrum of radioactive sources present in a sample; quantitative analyses are performed by counting and measuring the energy of individual photons emitted from radioactive elements present in the sample (20). Currently, the use of high resolution HPGe detectors in gamma spectrometry is the most common strategy for the identification and quantification of gamma emitting radionuclides in samples (20). By comparing the results from gamma spectrometry with those of a clinical PET/CT scanner for two cell lines (LK0412 and LK0626), the current study demonstrated that the differences in uptake values between radiated cells and controls were comparable between the two methods. The slightly higher variance of the quantitative results obtained with PET/CT indicated that the reconstruction correction factors for attenuation, scatter and the specific measurement geometry with low background need to be further evaluated and optimized for reconstruction parameter settings, scatter and attenuation correction strategies. A significant association for individual cell measurements between the two methods would be a strong indicator for the accurate comparison between gamma spectrometry and PET/CT measurements, the results of which may improve the evaluation process for batch measurements. A clinical PET/CT scanner may use up to 360 Petri dishes to produce a single PET acquisition of $4 \mathrm{~min}$. For a GS or a scintillation detector, this would be time consuming and cumbersome, as the accumulated acquisition time would be $>720$ min and the measurements would need to account for activity decay and variation in count statistics due to the short half-life of $109.7 \mathrm{~min}$ for ${ }^{18} \mathrm{~F}$. The use of a PET/CT scanner also aids the translation of data from in vitro cell studies to the clinical assessment of individual treatment responses in vivo. The sensitivity and specificity of $18 \mathrm{~F}-\mathrm{FDG}-\mathrm{PET}$ in a clinical setting is well established and have been shown to be $>90 \%$ for a number of investigation and lesion sizes $(21,22)$. Investigations and optimization of the PET acquisition settings, reconstruction parameters and correction methods for the Petri dish matrix will be required to further improve results with 
increased accuracy and less variation. In the present study, the standard OSEM reconstruction algorithm with PSF and TOF corrections was used but without any attenuation correction. Novel, more advanced reconstruction methods with improved signal-to-noise ratios such as Bayesian penalized likelihood reconstruction was also tested in the present study, but induced a higher variability in the measurements and streak artifacts in the image when attenuation correction was applied. Further studies are required to optimize and standardize acquisition and reconstruction parameter settings for batch measurements of in vitro samples with a clinical PET/CT system.

The results of a previous study indicated that a high maximum standardized uptake value (SUVmax) of FDG from PET/CT measurements predicts a poor prognosis in laryngeal cancer (23). Furthermore, the study revealed an association between high SUVmax and an increased expression of GLUT1, hypoxia-inducible factor-1 $\alpha$ (HIF-1 $\alpha$ ), PI3K and phosphorylated-AKT. Yamada et al (24) also demonstrated that the uptake of FDG was associated with GLUT1 and HIF-1 $\alpha$ expression in early stage oral squamous cell carcinomas. However, it has also been revealed that $18 \mathrm{~F}-\mathrm{FDG}$ uptake in HNSCC xenografts might not reflect the level of metabolic activity (hexokinase II and thymidine kinase-1 protein levels) (25).

The current study utilized three cell lines with different intrinsic radiation sensitivities and three cell lines with different intrinsic cetuximab sensitivities $(13,14,17)$. The results revealed that after radiotherapy and cetuximab treatment, a decrease in 18F-FDG uptake was observed, which was most prominent in the most sensitive cell lines LK0626, LK0412 and UT-SCC-14. It has been reported that the SUVmax is associated with the effectiveness of neoadjuvant chemoradiotherapy in oral squamous cell carcinoma (26). Furthermore, an increased sensitivity to radiation has been associated with GLUT1 downregulation in laryngeal carcinoma xenografts; however, no significant association between GLUT1 or HIF-1 $\alpha$ expression and 18F-FDG uptake was found (27). The present study identified a significant increase in GLUT1 mRNA expression following $24 \mathrm{~h}$ exposure to cetuximab in the sensitive cell line UT-SCC-14, which was not associated with increased 18F-FDG uptake. In HNSCC tumors, GLUT1 has been revealed to be directly associated with the degree of hypoxia (28). A previous study recently determined that hypoxia and HIF-1 $\alpha$ impact the sensitivity to cetuximab in HNSCC (29). However, the connection to GLUT1 and FDG uptake requires further elucidation.

To clarify the impact of FDG uptake on cell responses to cetuximab treatment, in vivo investigations must be performed. In vivo models combined with metabolic, pre-clinical small animal imaging will provide an increased possibility to translate results from in vitro models into clinical practice. In contrast to cell or tissue culture-based experiments, animal studies incorporate interacting physiological factors present in a complex living system, thereby providing quantitative, spatially and temporally-indexed information on normal and diseased tissues to monitor disease progression or treatment response over a period of time (30). Our in vitro results are a first step to determine if GS and PET/CT analysis can be used for evaluating treatment response. In the future, treatment response evaluation may be important in determining personalized treatments for patients with HNSCC.
In summary, the current study developed a novel and reliable method for the measurement of intracellular 18F-FDG in HNSCC cell lines. Furthermore, it was determined that PET/CT may be used for the measurement of high sample numbers with a satisfactory result compared with gamma spectrometry. The results also indicated that 18F-FDG uptake can be assessed in an in vitro model via gamma spectrometry and a clinical PET/CT system and has an association with cetuximab and radiation treatment responses.

\section{Acknowledgements}

The authors would like to thank Professor Reidar Grenman (University of Turku, Turku, Finland) for providing the UT-SCC cell lines.

\section{Funding}

This study was supported by The Swedish Cancer Society (grant nos. 2010/545 and 2017/301), ALF Grants, Region Östergötland and the Research Funds of Linköping University Hospital.

\section{Availability of data and materials}

The datasets used and/or analyzed during the current study are available from the corresponding author on reasonable request.

\section{Authors' contributions}

KR and MR designed the experiment. NM, MR, EW and KR performed the experiment. NM, EW and MR analyzed the data. NM, MR, EW and KR wrote the manuscript. All authors read and approved the final manuscript.

\section{Ethics approval and consent to participate}

This study has been performed under general approval from the Swedish Radiation Safety Authority allowing Linköping University Hospital to handle and use radioactive substances (approval no. Cm-016-10143 SSM 2016-4467). Collection of normal oral tissue was approved by the Linköping University Ethical Committee (approval no. M156-05). Signed informed consent was obtained from all the patients.

\section{Patient consent for publication}

Not applicable.

\section{Competing interests}

The authors declare that they have no competing interests.

\section{References}

1. Ferlay J, Soerjomataram I, Dikshit R, Eser S, Mathers C, Rebelo M, Parkin DM, Forman D and Bray F: Cancer incidence and mortality worldwide: Sources, methods and major patterns in GLOBOCAN 2012. Int J Cancer 136: E359-E386, 2015.

2. Seiwert TY, Salama JK and Vokes EE: The chemoradiation paradigm in head and neck cancer. Nat Clin Pract Oncol 4: 156-171, 2007. 
3. Dassonville O, Formento JL, Francoual M, Ramaioli A, Santini J, Schneider M, Demard F and Milano G: Expression of epidermal growth factor receptor and survival in upper aerodigestive tract cancer. J Clin Oncol 11: 1873-1878, 1993.

4. Bonner JA, Harari PM, Giralt J, Azarnia N, Shin DM, Cohen RB, Jones CU, Sur R, Raben D, Jassem J, et al: Radiotherapy plus cetuximab for squamous-cell carcinoma of the head and neck. N Engl J Med 354: 567-578, 2006

5. Wu W and Zhao S: Metabolic changes in cancer: Beyond the Warburg effect. Acta Biochim Biophys Sin (Shanghai) 45: 18-26, 2013.

6. Yamamoto M, Inohara $\mathrm{H}$ and Nakagawa T: Targeting metabolic pathways for head and neck cancers therapeutics. Cancer Metastasis Rev 36: 503-514, 2017.

7. Beloueche-Babari M, Box C, Arunan V, Parkes HG, Valenti M, De Haven Brandon A, Jackson LE, Eccles SA and Leach MO: Acquired resistance to EGFR tyrosine kinase inhibitors alters the metabolism of human head and neck squamous carcinoma cells and xenograft tumours. Br J Cancer 112: 1206-1214, 2015.

8. Kircher MF, Hricak H and Larson SM: Molecular imaging for personalized cancer care. Mol Oncol 6: 182-195, 2012.

9. Baschnagel AM, Wobb JL, Dilworth JT, Williams L, Eskandari M, Wu D, Pruetz BL and Wilson GD: The association of (18)F-FDG PET and glucose metabolism biomarkers GLUT1 and HK2 in p16 positive and negative head and neck squamous cell carcinomas. Radiother Oncol 117: 118-124, 2015.

10. Carvalho KC, Cunha IW, Rocha RM, Ayala FR, Cajaíba MM Begnami MD, Vilela RS, Paiva GR, Andrade RG and Soares FA: GLUT1 expression in malignant tumors and its use as an immunodiagnostic marker. Clinics (Sao Paulo) 66: 965-972, 2011.

11. Kunkel M, Reichert TE, Benz P, Lehr HA, Jeong JH, Wieand S, Bartenstein P, Wagner W and Whiteside TL: Overexpression of Glut-1 and increased glucose metabolism in tumors are associated with a poor prognosis in patients with oral squamous cell carcinoma. Cancer 97: 1015-1024, 2003.

12. Wang YD, Li SJ and Liao JX: Inhibition of glucose transporter 1 (GLUT1) chemosensitized head and neck cancer cells to cisplatin. Technol Cancer Res Treat 12: 525-535, 2013.

13. Jedlinski A, Ansell A, Johansson AC and Roberg K: EGFR status and EGFR ligand expression influence the treatment response of head and neck cancer cell lines. J Oral Pathol Med 42: 26-36, 2013.

14. Jerhammar F, Johansson AC, Ceder R, Welander J, Jansson A, Grafström RC, Söderkvist P and Roberg K: YAP1 is a potential biomarker for cetuximab resistance in head and neck cancer. Oral Oncol 50: 832-839, 2014

15. Roberg K, Ceder R, Farnebo L, Norberg-Spaak L and Grafstrom RC: Multiple genotypic aberrances associate to terminal differentiation-deficiency of an oral squamous cell carcinoma in serum-free culture. Differentiation 76: 868-880, 2008.

16. Leutenegger CM, Mislin CN, Sigrist B, Ehrengruber MU, Hofmann-Lehmann R and Lutz H: Quantitative real-time PCR for the measurement of feline cytokine mRNA. Vet Immunol Immunopathol 71: 291-305, 1999.

17. Jedlinski A, Garvin S, Johansson AC, Edqvist PH, Ponten F and Roberg K: Cetuximab sensitivity of head and neck squamous cell carcinoma xenografts is associated with treatment-induced reduction in EGFR, pEGFR, and pSrc. J Oral Pathol Med 46: 717-724, 2017.
18. Hentschel M, Paulus T, Mix M, Moser E, Nitzsche EU and Brink I: Analysis of blood flow and glucose metabolism in mammary carcinomas and normal breast: A H2(15)O PET and 18F-FDG PET study. Nucl Med Commun 28: 789-797, 2007.

19. Machtay M, Natwa M, Andrel J, Hyslop T, Anne PR, Lavarino J, Intenzo CM and Keane W: Pretreatment FDG-PET standardized uptake value as a prognostic factor for outcome in head and neck cancer. Head Neck 31: 195-201, 2009.

20. Orabi H, Al-Shareaif A and El Galefi M: Gamma-ray measurements of naturally occurring radioactive sample from Alkharje City. J Radioanal Nucl Chem 269: 99-102, 2006.

21. Behzadi A, Ung Y, Lowe V and Deschamps C: The role of positron emission tomography in the management of non-small cell lung cancer. Can J Surg 52: 235-242, 2009.

22. Gould MK, Maclean CC, Kuschner WG, Rydzak CE and Owens DK: Accuracy of positron emission tomography for diagnosis of pulmonary nodules and mass lesions: A meta-analysis. JAMA 285: 914-924, 2001.

23. Zhao K, Yang SY, Zhou SH, Dong MJ, Bao YY and Yao HT: Fluorodeoxyglucose uptake in laryngeal carcinoma is associated with the expression of glucose transporter-1 and hypoxia-inducible-factor- $1 \alpha$ and the phosphoinositide 3-kinase/protein kinase B pathway. Oncol Lett 7: 984-990, 2014.

24. Yamada T, Uchida M, Kwang-Lee K, Kitamura N, Yoshimura T, Sasabe E and Yamamoto T: Correlation of metabolism/hypoxia markers and fluorodeoxyglucose uptake in oral squamous cell carcinomas. Oral Surg Oral Med Oral Pathol Oral Radiol 113: 464-471, 2012.

25. Mason NS, Lopresti BJ, Ruszkiewicz J, Dong X, Joyce S, Leef G, Sen M, Wahed AS, Mathis CA, Grandis JR and Thomas SM: Utility of 3'-[(18)F]fluoro-3'-deoxythymidine as a PET tracer to monitor response to gene therapy in a xenograft model of head and neck carcinoma. Am J Nucl Med Mol Imaging 3: 16-31, 2013.

26. Miyawaki A, Ikeda R, Hijioka H, Ishida T, Ushiyama M, Nozoe E and Nakamura N: SUVmax of FDG-PET correlates with the effects of neoadjuvant chemoradiotherapy for oral squamous cell carcinoma. Oncol Rep 23: 1205-1212, 2010.

27. Shen LF, Zhao X, Zhou SH, Lu ZJ, Zhao K, Fan J and Zhou ML: In vivo evaluation of the effects of simultaneous inhibition of GLUT-1 and HIF-1alpha by antisense oligodeoxynucleotides on the radiosensitivity of laryngeal carcinoma using micro 18F-FDG PET/CT. Oncotarget 8: 34709-34726, 2017.

28. Starska K, Forma E, Jozwiak P, Bryś M, Lewy-Trenda I, Brzezińska-Błaszczyk E and Krześlak A: Gene and protein expression of glucose transporter 1 and glucose transporter 3 in human laryngeal cancer-the relationship with regulatory hypoxia-inducible factor-1 $\alpha$ expression, tumor invasiveness, and patient prognosis. Tumour Biol 36: 2309-2321, 2015.

29. Wiechec E, Hansson KT, Alexandersson L, Jonsson JI and Roberg K: Hypoxia mediates differential response to anti-EGFR therapy in HNSCC cells. Int J Mol Sci 18: E943, 2017.

30. Ben-Haim S and Ell P: 18F-FDG PET and PET/CT in the evaluation of cancer treatment response. J Nucl Med 50: 88-99, 2009.

This work is licensed under a Creative Commons Attribution-NonCommercial-NoDerivatives 4.0 International (CC BY-NC-ND 4.0) License. 